\title{
MODELAGEM E SIMULAÇÃO DE CURVAS DE RUPTURA DE ATIVIDADE QUITOSANOLÍTICA PRODUZIDA POR Paenibacillus ehimensis USANDO ADSORÇÃO EM LEITO EXPANDIDO
}

\author{
PADILHA, C. E. A. ${ }^{1}$; OLIVEIRA, J. A. ${ }^{1}$; MACEDO, G. R. ${ }^{1}$; SANTOS, E. S. ${ }^{1}$
}

${ }^{1}$ Laboratório de Engenharia Bioquímica, Departamento de Engenharia Química,Universidade Federal do Rio Grande do Norte (UFRN)

\begin{abstract}
RESUMO - Neste estudo é apresentada a modelagem matemática da adsorção de quitosanases produzidas por Paenibacillus ehimensis usando uma coluna de leito expandido. As corridas de adsorção foram realizadas em coluna de vidro $30,00 \mathrm{~cm} \mathrm{x}$ $2,64 \mathrm{~cm}$ e a Streamline DEAE foi usado como adsorvente. Os experimentos foram executados em condições diferentes de concentração inicial $(0,20$ a $0,26 \mathrm{UA} / \mathrm{mL})$, altura do leito fixo $(5,0$ e $7,5 \mathrm{~cm})$ e velocidade de fluxo $(80$ a $240 \mathrm{~cm} / \mathrm{h})$. O modelo de taxa geral desenvolvido levou em consideração a transferência de massa, a adsorção, os efeitos hidrodinâmicos e a difusão no poro. O algoritmo metaheurístico PSO (particle swarm optimization) foi introduzido para estimar parâmetros cinéticos e de transferência de massa, propiciando maior ajuste do modelo. As simulações mostraram boa concordância com as curvas de ruptura experimentais, alcançando $S S R$ de até 0,265, para os graus de expansão 1,00-2,60.
\end{abstract}

\section{INTRODUÇÃO}

As quitosanases (EC 3.2.1.132) são enzimas hidrolíticas que atuam nas ligações glicosídicas dos polímeros quitosana e quitina produzindo quitooligossacarídeos (QOS), e são expressas por uma variedade de micro-organismos, em sua maioria bactérias (Sun et al., 2007; Gao et al., 2008). Atualmente, já se conhece uma diversidade de benefícios relativos ao uso dos QOS devido a sua ação antitumoral (Shen et al., 2009), prebiótica (Liang et al., 2013), antibacteriana (Xia et al., 2010). Um dos problemas da aplicação efetiva das enzimas microbianas, inclusive das quitosanases, é o alto custo envolvido na recuperação e purificação destes produtos.

A adsorção em leito expandido (ALE) é uma técnica integrativa que combina clarificação, recuperação e purificação parcial em uma única etapa, se valendo de conceitos de cromatografia e fluidização de sólidos. A alimentação da ALE pode ser efetuada com extrato bruto não tratado, uma vez que o fluxo ascendente promove a segregação das partículas adsorventes, evitando a 
colmatação da coluna (Silveira et al., 2009). A estabilidade da expansão do leito aproxima a ALE das características da coluna em leito fixo.

O desempenho do processo de adsorção em coluna pode ser avaliado pelas curvas de ruptura. Neste gráfico são registrados a concentração do efluente após a passagem na coluna e o tempo de corrida, revelando a saturação gradual do leito adsorvente pela molécula de interesse. A modelagem matemática da curva de ruptura torna-se, então, fundamental para entender o comportamento da adsorção e a otimização de parâmetros operacionais, sem a exigência de mais experimentos (Moraes et al., 2009; Yun et al., 2005).

Assim, o presente trabalho tem como enfoque a modelagem fenomenológica das curvas de ruptura de quitosanases usando a resina Streamline DEAE, um trocador aniônico. As quitosanases foram produzidas pela cepa Paenibacillus ehimensis e a coluna foi operada em leito fixo e leito expandido. No estudo buscou-se o ajuste das curvas de ruptura experimentais pelo auxílio da subrotina de estimação enxame de partículas (Particle Swarm Optimization, PSO).

\section{EXPERIMENTAL}

\subsection{Produção de quitosanases}

A cepa Paenibacillus ehimensis NRRL 23118 foi usada na produção de quitosanases. O concentrado celular, armazenado a $10 \% \mathrm{v} / \mathrm{v}$ glicerol, foi transferido a Erlenmeyers de 250 contendo $50 \mathrm{~mL}$ do meio A (1,0 g.L $\mathrm{L}^{-1}$ peptona, 1,0 g. $\mathrm{L}^{-1}$ extrato de levedura, $0,5 \mathrm{~g} . \mathrm{L}^{-1}$ sulfato de magnésio, 1,0 g. $\mathrm{L}^{-1}$ fosfato dibásico de potássio, 2,0 g.L $\mathrm{L}^{-1}$ glicose, $\mathrm{pH}$ 7,0) e incubados a $309 \mathrm{~K} \mathrm{e}$ $120 \mathrm{rpm}$ durante $24 \mathrm{~h}$. Alíquotas do meio A $(5 \mathrm{~mL})$ foram inoculadas em $45 \mathrm{~mL}$ do meio B $(6,0$ g. $\mathrm{L}^{-1}$ peptona, $0,5 \mathrm{~g} . \mathrm{L}^{-1}$ sulfato de magnésio, 1,0 g.L $\mathrm{L}^{-1}$ fosfato dibásico de potássio, 1,0 g.L $\mathrm{L}^{-1}$ glicose, 3,0 g. $\mathrm{L}^{-1}$ quitosana, $\mathrm{pH} 7,0$ ). A produção foi realizada em shaker durante $48 \mathrm{~h}$, nas mesmas condições do cultivo anterior (Araújo et al., 2013). O produto foi armazenado em freezer a $253 \mathrm{~K}$, acrescido de $0,05 \%$ de azida de sódio.

\subsection{Coluna e adsorvente}

Uma coluna de vidro com diâmetro $2,64 \mathrm{~cm}$ e altura de $30,00 \mathrm{~cm}$ foi confeccionada para operar em leito expandido. Um pistão móvel foi utilizado para permitir diferentes graus de expansão do leito e o distribuidor consistia de um leito de microesferas de vidro com altura de 3,0 $\mathrm{cm}$. A alimentação da coluna foi realizada por uma bomba peristáltica (Perimax 12, Spetec). A resina utilizada nos ensaios de adsorção foi a Streamline DEAE (GE Healthcare, Uppsala, Sweden), um trocador aniônico da primeira geração de adsorventes próprios para adsorção em leito expandido.

\subsection{Purificação de quitosanases}

Em cada ensaio a coluna foi equilibrada com tampão Fosfato 50,0 mM e pH 8,0 e, em seguida, fez-se passar 200,0 mL de caldo fermentado clarificado com $\mathrm{pH} 8,0$. As alturas do leito fixo de 5,0 e $7,5 \mathrm{~cm}$ foram selecionadas e as velocidades de fluxo variaram de $80 \mathrm{~cm} / \mathrm{h}$ a 240 $\mathrm{cm} / \mathrm{h}$. As corridas foram realizadas em leito fixo $\left(\mathrm{H} / \mathrm{H}_{0}=1\right)$ e em leito expandido $\left(\mathrm{H} / \mathrm{H}_{0}=1,80\right.$, $\mathrm{H} / \mathrm{H}_{0}=2,20$ e $\left.\mathrm{H} / \mathrm{H}_{0}=2,60\right)$ na temperatura de $25^{\circ} \mathrm{C}$. A concentração de atividade quitosanolítica 
foi analisada na alimentação e na saída da coluna.

\subsection{Determinação da atividade quitosanolítica}

A atividade enzimática foi determinada através dos passos realizados em Araújo et al. (2013). A formação de açúcares redutores foi analisada pelo método DNS (Miller, 1959), usando D-glicosamina (Sigma Aldrich, Ohio, USA) como padrão. Uma unidade de atividade de quitosanase (1 UA) foi definida como a quantidade de enzima capaz de formar $1 \mu \mathrm{mol}$ de Dglicosamina por minuto nas condições estabelecidas.

\section{MODELO MATEMÁTICO}

O desenvolvimento do modelo matemático para o processo de adsorção de quitosanases em colunas de leito fixo e de leito expandido necessitou da definição de algumas hipóteses:

i. O adsorvente foi considerado como uma esfera de densidade uniforme e grupos funcionais igualmente distribuídos. O tamanho das partículas seguiu uma distribuição axial definida por Tong e Sun (2002). O leito adsorvente apresentou empacotamento homogêneo.

ii. $\mathrm{O}$ adsorvente foi adotado como um material poroso, no qual o adsorbato pode difundir-se livremente. Representou-se a difusão intraparticular pelo termo de difusividade efetiva $\left(D_{e f}\right)$.

iii. O comportamento hidrodinâmico da fase líquida foi representado pelo modelo de dispersão axial.

iv. A transferência de massa na superfície do adsorvente foi governada pelo coeficiente de transferência no filme líquido $\left(k_{f}\right)$.

v. O equilíbrio de adsorção foi representado pelo modelo de Langmuir como visto na Equação 1, onde a concentração de atividade enzimática no poro $\left(c^{*}\right)$ está em equilíbrio local com a atividade retida nas paredes do adsorvente $\left(q^{*}\right)$. Os parâmetros $\lambda$ e $1 / b$ são a capacidade máxima de adsorção das quitosanases e o coeficiente de adsorção, respectivamente.

$$
q^{*}=\frac{\lambda b c^{*}}{1+b c^{*}}
$$

O balanço de massa na fase fluida em um elemento de volume da coluna pode ser expresso pela Equação 2.

$$
\begin{aligned}
& \frac{\partial c}{\partial t}=D_{a x} \frac{\partial^{2} c}{\partial z^{2}}-\frac{u}{\varepsilon} \frac{\partial c}{\partial z}-\frac{3 k_{f}(1-\varepsilon)\left(c-c_{f}\right)}{\varepsilon R_{i}} \\
& t=0 ; \quad c(z, 0)=0 \text { para } 0 \leq z \leq H \\
& z=0 ; \quad c=c_{0}+\frac{(1-\varepsilon) D_{a x}}{u} \frac{\partial c}{\partial z} \quad \text { para } t>0
\end{aligned}
$$




$$
z=H ; \quad \frac{\partial c}{\partial z}=0 \text { para } t>0
$$

$\mathrm{Na}$ fase sólida, a dispersão se insere em termos da quantidade de atividade enzimática adsorvida, gerando a Equação 6 (Wright e Glasser, 2001; Tong et al., 2003).

$$
\begin{aligned}
& (1-\varepsilon) \frac{\partial \bar{q}}{\partial t}=D_{s} \frac{\partial^{2} \bar{q}}{\partial z^{2}}+\frac{3 k_{f}(1-\varepsilon)\left(c-c_{f}\right)}{R_{i}} \\
& t=0 ; \quad \bar{q}(z, 0)=0 \text { para } 0 \leq z \leq H \\
& z=0 ; \quad \frac{\partial \bar{q}}{\partial z}=0 \text { para } t>0 \\
& z=H ; \frac{\partial \bar{q}}{\partial z}=0 \text { para } t>0
\end{aligned}
$$

Quando se utiliza adsorventes porosos em processos de adsorção é importante a inclusão do modelo de dispersão do poro (PDM) (Wright e Glasser, 2001; Tong et al., 2003; Yun et al., 2005), conforme a Equação 10.

$$
\begin{aligned}
& \varepsilon_{p} \frac{\partial c_{i}}{\partial t}=\frac{\partial q_{i}}{\partial t}+\varepsilon_{p} D_{e f}\left(\frac{\partial^{2} c_{i}}{\partial r^{2}}+\frac{2}{r} \frac{\partial c_{i}}{\partial r}\right) \\
& t=0 ; c=c_{0} ; c_{i}=0 ; q_{i}=0 \\
& r=0 ; \frac{\partial c_{i}}{\partial r}=0 \text { para } t>0 \\
& r=R ; \frac{\partial c_{i}}{\partial z}=\frac{R}{3 \varepsilon_{p} D_{e f}} \frac{\partial \bar{q}}{\partial t} \text { para } t>0
\end{aligned}
$$

A otimização por enxame de partículas é um algoritmo de busca estocástico inspirado na movimentação de pássaros em busca de alimento. Cada elemento ou partícula do enxame procura a melhor solução baseado na melhor posição encontrada por ele próprio e pelo conjunto de partículas. As partículas se movem pelo intervalo de busca e trocam informações com as outras partículas, de acordo com as Equações 14 e 15 (Burkert et al., 2011).

$$
v_{p, d}^{k+1}=w \cdot v_{p, d}^{k}+c_{1} \cdot r_{1}\left(x_{p, d}^{i n d}-x_{p, d}^{k}\right)+c_{2} \cdot r_{2}\left(x_{d}^{g l o b}-x_{p, d}^{k}\right)
$$




$$
x_{p, d}^{k+1}=x_{p, d}^{k}+v_{p, d}^{k+1}
$$

Nas Equações 14 e 15, $p$ representa a partícula, $d$ é a direção de busca, $k$ é o número de iterações, $v$ é a velocidade da partícula, $x$ é a posição da partícula, $x^{i n d}$ é a melhor posição da partícula e $x^{g l o b}$ é a melhor posição encontrada pelo enxame. Os termos $r_{1}$ e $r_{2}$ são dois números randômicos que se distribuem entre 0 e 1 . Os parâmetros $w, c_{1}$ e $c_{2}$ tratam-se do peso inercial, parâmetro de cognição e parâmetro social, respectivamente.

As simulações do modelo de taxa geral foram realizadas usando a linguagem Fortran, no software Microsoft Visual Studio 2010. O método de diferenças finitas foi usado para as discretizações nas coordenadas espaciais $z$ e $r$, sendo adotados trinta pontos em cada caso. Os balanços diferenciais descritos nas Equações 2, 6 e 10 foram resolvidos pela rotina DASSL IMSL. No algoritmo PSO foram usadas 20 partículas e 20 iterações; o peso inercial e os parâmetros de cognição e social foram fixados em $0,7,1,0$ e 1,0, respectivamente. Os intervalos de busca do PSO para os parâmetros do modelo foram: $\varepsilon \in[0,5 ; 0,9], D_{a x} \in\left[1.10^{-9} ; 1.10^{-4}\right], D_{s} \in\left[1.10^{-9} ; 1.10^{-4}\right], k_{f} \in$ $\left[1.10^{-12} ; 1.10^{-6}\right], D_{e f} \in\left[1.10^{-12} ; 1.10^{-6}\right], \lambda \in[0,1 ; 50], b \in[0,1 ; 10]$.

\section{RESULTADOS E DISCUSSÕES}

O uso de correlações para determinação de parâmetros cinéticos e hidrodinâmicos é uma prática bem comum na modelagem de processos de adsorção, entretanto, há situações em que esta prática torna-se falha, como, por exemplo, a aplicação de extrato bruto para adsorção para quitosanases (Moraes et al., 2009). Para tanto, o emprego de uma rotina de estimação de parâmetros (como o PSO) é importante para contornar estes obstáculos.

A Figura 1 mostra as curvas de ruptura experimentais e simuladas, além das variáveis operacionais: concentração de alimentação, altura do leito sedimentado e velocidade de fluxo. As curvas de ruptura experimentais diferem entre si, principalmente por causa da velocidade de fluxo, e se assemelham a uma função sigmoidal ( $S$-shape). Os parâmetros do modelo estimados pelo $P S O$ estão dispostos na Tabela 1 e foram usados para simular a adsorção das quitosanases. Nota-se pela Tabela 1 que o estimador também conseguiu reconhecer as tendências de alguns parâmetros como a dispersão axial, o coeficiente de transferência de massa, a capacidade máxima de adsorção, a constante de adsorção e, principalmente, a porosidade do leito.

Os dados experimentais e simulados foram comparados mediante a soma quadrática residual $(S S R)$, conforme a Equação 16. Através deste cálculo observa-se uma boa concordância entre as curvas experimentais e simuladas, apesar do afastamento considerável nas regiões $\mathrm{C} / \mathrm{C}_{0}<0,2$. Os valores de $S S R$ foram iguais a $0,461,0,265,0,487$ e 0,373 para os casos $\mathrm{A}, \mathrm{B}, \mathrm{C}$ e $\mathrm{D}$, respectivamente.

$$
S S R=\left(C / C_{0}{ }^{\exp }-C / C_{0}{ }^{\text {calc }}\right)^{2}
$$

Outro paralelo entre as curvas de ruptura experimentais e simuladas foi realizado a partir da eficiência do processo, como se pode observar na Equação 17. A Tabela 2 mostra os valores da eficiência do processo provenientes das curvas de ruptura experimentais e simuladas nas quatro situações testadas. Neste trabalho a eficiência do processo foi calculada quando a concentração de 
saída atingiu $15 \%$ da concentração inicial. Nos casos do leito fixo (A) e do leito expandido na velocidade $160 \mathrm{~cm} / \mathrm{h}$ (C) a função eficiência experimental e calculada foram próximas, nos casos $\mathrm{B}$ e D, em contrapartida, elas foram bem diferentes. Este afastamento foi causado provavelmente por instabilidades na expansão do leito durante o ínicio das corridas, o que provocou saídas $\mathrm{C} / \mathrm{C}_{0}$ mais elevadas do que na curva simulada.

$$
\phi_{15 \%}=\frac{\left(0,15 \cdot t_{C / C_{0}=0,15}-\int_{0}^{t} C / C_{0}=0,15 C / C_{0} \cdot d t\right)}{0,15 \cdot t_{C / C_{0}=0,15}} \cdot 100
$$
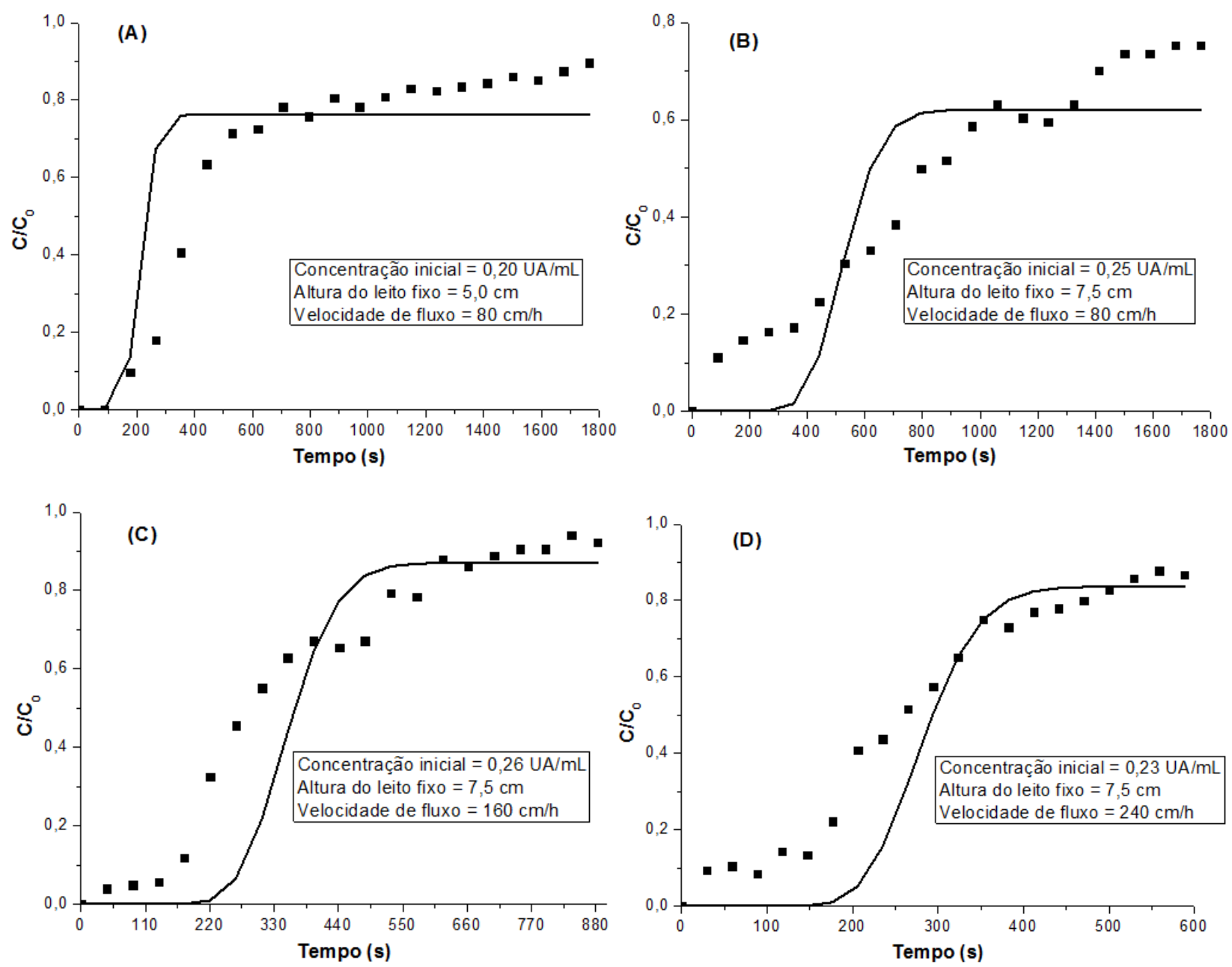

Figura 1 - Curvas de ruptura da adsorção de quitosanases em leito fixo (A) e expandido (B, C e D). Os pontos (ロ) são os dados experimentais. As linhas contínuas (-) são os dados simulados. 
Tabela 1 - Parâmetros estimados pelo algoritmo PSO nas quatro simulações

\begin{tabular}{|c|c|c|c|c|}
\hline Parâmetros & Simulação A & Simulação B & Simulação C & Simulação D \\
\hline$\varepsilon_{\mathrm{L}}$ & 0,549 & 0,628 & 0,809 & 0,832 \\
\hline $\mathrm{D}_{\mathrm{ax}}\left(10^{-5} \mathrm{~cm}^{2} / \mathrm{s}\right)$ & 0,446 & 1,198 & 0,260 & 6,466 \\
\hline $\mathrm{k}_{\mathrm{L}}\left(10^{-6} \mathrm{~cm} / \mathrm{s}\right)$ & 1,618 & 5,835 & 5,271 & 9,648 \\
\hline $\mathrm{D}_{\mathrm{sol}}\left(10^{-8} \mathrm{~cm} / \mathrm{s}\right)$ & 3,104 & 20,735 & 3,805 & 9,655 \\
\hline $\mathrm{D}_{\mathrm{ef}}\left(10^{-8} \mathrm{~cm}^{2} / \mathrm{s}\right)$ & 6,244 & 20,912 & 1,117 & 8,810 \\
\hline$\lambda(\mathrm{UA} / \mathrm{g})$ & 26,661 & 25,272 & 29,703 & 36,014 \\
\hline$b(\mathrm{~mL} / \mathrm{UA})$ & 4,301 & 3,092 & 4,996 & 4,493 \\
\hline
\end{tabular}

Tabela 2 - Eficiência experimental e calculada para a adsorção de quitosanases em colunas de leito fixo e expandido.

\begin{tabular}{|c|c|c|c|c|}
\hline Eficiência & A & B & C & D \\
\hline$\phi_{15 \%}^{\exp }(\%)$ & 72,85 & 39,62 & 61,59 & 33,79 \\
\hline$\phi_{15 \%}^{\text {calc }}(\%)$ & 75,59 & 87,62 & 64,58 & 88,94 \\
\hline
\end{tabular}

\section{CONCLUSÃO}

O objetivo do trabalho foi elaborar um modelo de taxa geral para simular as curvas de ruptura de quitosanases produzidas por Paenibacillus ehimensis em Streamline DEAE, se valendo da ferramenta de estimação de parâmetros PSO. Como desejado, as curvas de ruptura experimentais demonstraram um comportamento semelhante a uma sigmóide, o que facilitou a tarefa do modelo. O estimador $P S O$ organizou adequadamente cada conjunto de parâmetros a uma situação específica, possibilitando curvas de ruptura simuladas próximas das curvas de ruptura experimentais. Enfim, mostra-se válido o uso de rotinas de estimação na modelagem matemática de sistemas ALE para recuperação de quitosanases.

\section{REFERÊNCIAS}

ARAÚJO, N. K.; ASSIS, C. F.; SANTOS, E. S.; MACEDO, G. R.; FARIAS, L. F.; JÚNIOR, H. A.; PEDROSA, M. F. F.; PAGNONCELLI, M. G. B. Production of Enzymes by Paenibacillus chitinolyticus and Paenibacillus ehimensis to Obtain Chitooligosaccharides. Applied Biochemistry and Biotechnology, v. 170, p. 292-300, 2013.

BURKERT, C. A. V.; BARBOSA, G. N. O.; MAZUTTI, M. A.; MAUGERI, F. Mathematical modeling and experimental breakthrough curves of cephalosporin $\mathrm{C}$ adsorption in a fixed-bed column. Process Biochemistry, v. 46, p. 1270-1277, 2011.

GAO, X. -A; JU, W. -T.; JUNG, W. -J.; PARK, R. -D. Purification and characterization of chitosanase from Bacillus cereus D-11. Carbohydrate Polymers, v. 72, p. 513-520, 2008.

LIANG, T. -W.; LIU, C. -P.; WU, C.; WANG, S. -L. Applied development of crude enzyme from Bacillus cereus in prebiotics and microbial community changes in soil. Carbohydrate Polymers, v. 92, p. 2141-2148, 2013.

MILLER, G. L. Use of dinitrosalicylic acid reagent for determination of reducing Sugar. Anal. Chem, v. 31, 3, p. 426-428, 1959. 
SHEN, K. -T.; CHEN, M. -H.; CHAN, H. -Y.; JENG, J. -H.; WANG, Y. -J. Inhibitory effects of chitooligosaccharides on tumor growth and metastasis. Food and Chemical Toxicology, v. 47, p. 1864-1871, 2009.

SILVEIRA, E.; SOUZA-JÚNIOR, M. E.; SANTANA, J. C. C.; CHAVES, A. C.; PORTO, A. L. F.; TAMBOURGI, E. B. Expanded bed adsorption of bromelain (E.C. 3. 4.22.33) from Ananas comosus crude extract. Brazilian Journal of Chemical Engineering, v. 26, 2009.

SUN, Y.; HAN, B.; LIU, W.; ZHANG, J.; GAO, X. Substrate induction and statistical optimization for the production of chitosanase from Microbacterium sp. OU01. Bioresource Technology, v. 98, p. 1548-1553, 2007.

TONG, X. -D.; SUN, Y. Particle size and density distributions of two dense matrices in an expanded bed system. Journal of Chromatography A, v. 977, p. 173-183, 2002.

TONG, X. -D.; XUE, D.; SUN, Y. Modeling of expanded bed protein adsorption by taking into account the axial particle size distribution. Biochemical Engineering Journal, v. 16, p. 265-272, 2003.

WRIGHT, P. R.; GLASSER, B. J. Modeling Mass Transfer and Hydrodynamics in Fluidized-Bed Adsorption of Proteins. AIChE Journal, v. 47, 474-488, 2001.

XIA, W. S.; LIU, P.; ZHANG, J. L.; CHEN, J. Biological activities of chitosan and chitooligossacharides. Food Hydrocolloids, v. 25, p. 170-179, 2010.

YUN, J.; LIN, Q. -X.; YAO, S. -J. Predictive modeling of protein adsorption along the bed height by taking into account the axial nonuniform liquid dispersion and particle classification in expanded beds. Journal of Chromatography A, v. 1095, p. 16-26, 2005. 